\title{
Homeostatic and pathogenic extramedullary hematopoiesis
}

This article was published in the following Dove Press journal:

Clinical Ophthalmology

20 March 2010

Number of times this article has been viewed

\section{Chang $\mathrm{H} \mathrm{Kim}$}

Laboratory of Immunology and Hematopoiesis, Department of Comparative Pathobiology; Purdue Cancer Center, Purdue University, West Lafayette, IN, USA
Correspondence: Chang H Kim

Department of Comparative Pathobiology, 725 Harrison Street, Purdue University,

West Lafayette, IN 47907, USA

Tel +I 7654940976

Fax + I 7654949830

Email chkim@purdue.edu
Abstract: Extramedullary hematopoiesis $(\mathrm{EH})$ is defined as hematopoiesis occurring in organs outside of the bone marrow; it occurs in diverse conditions, including fetal development, normal immune responses, and pathological circumstances. During fetal development, before formation of mature marrow, EH occurs in the yolk sac, fetal liver, and spleen. EH also occurs during active immune responses to pathogens. Most frequently, this response occurs in the spleen and liver for the production of antigen-presenting cells and phagocytes. EH also occurs when the marrow becomes inhabitable for stem and progenitor cells in certain pathological conditions, including myelofibrosis, where marrow cells are replaced with collagenous connective tissue fibers. Thus, EH occurs either actively or passively in response to diverse changes in the hematopoietic environment. This article reviews the key features and regulators of the major types of EH.

Keywords: myelopoiesis, cytokines, myeloid derived suppressor cells, T cells, infection, dendritic cells, FoxP3, myelofibrosis

\section{Introduction}

Extramedullary hematopoiesis (EH) refers to the hematopoiesis that occurs in organs other than bone marrow. A classic example of EH is the increased ectopic erythropoiesis in a liver or spleen in hypoxia due to increased erythropoietin production. ${ }^{1} \mathrm{EH}$ can be further classified into active or passive categories. Normal hematopoiesis, which occurs in the fetal yolk sac, liver, and spleen, is an example of active EH; it is programmed as an essential process for routine fetal development. Another example of active $\mathrm{EH}$ is that which occurs in the spleen and liver during immune responses following infection. By contrast, EH also occurs as a result of failed marrow hematopoiesis in peripheral organs such as the liver and spleen and this is considered a passive form of EH. Both active and passive EH produce blood cells such as antigen-presenting cells, granulocytes, NK cells, red blood cells, and/or platelets for the growth or survival of the host. Inadequate EH leads to insufficient production or maturation of blood cells, while excessive EH leads to inflammatory diseases (Figure 1). This article will review the key aspects of major types of EH.

\section{Factors required for hematopoiesis}

Hematopoiesis is a complex process regulated by multiple factors. Hematopoiesis occurs in specialized tissue sites (eg, "hematopoietic niches") conducive for the maintenance and differentiation of stem and progenitor cells. For example, hematopoietic stem cells, which are quiescent and self-renewing, are present in the marrow 


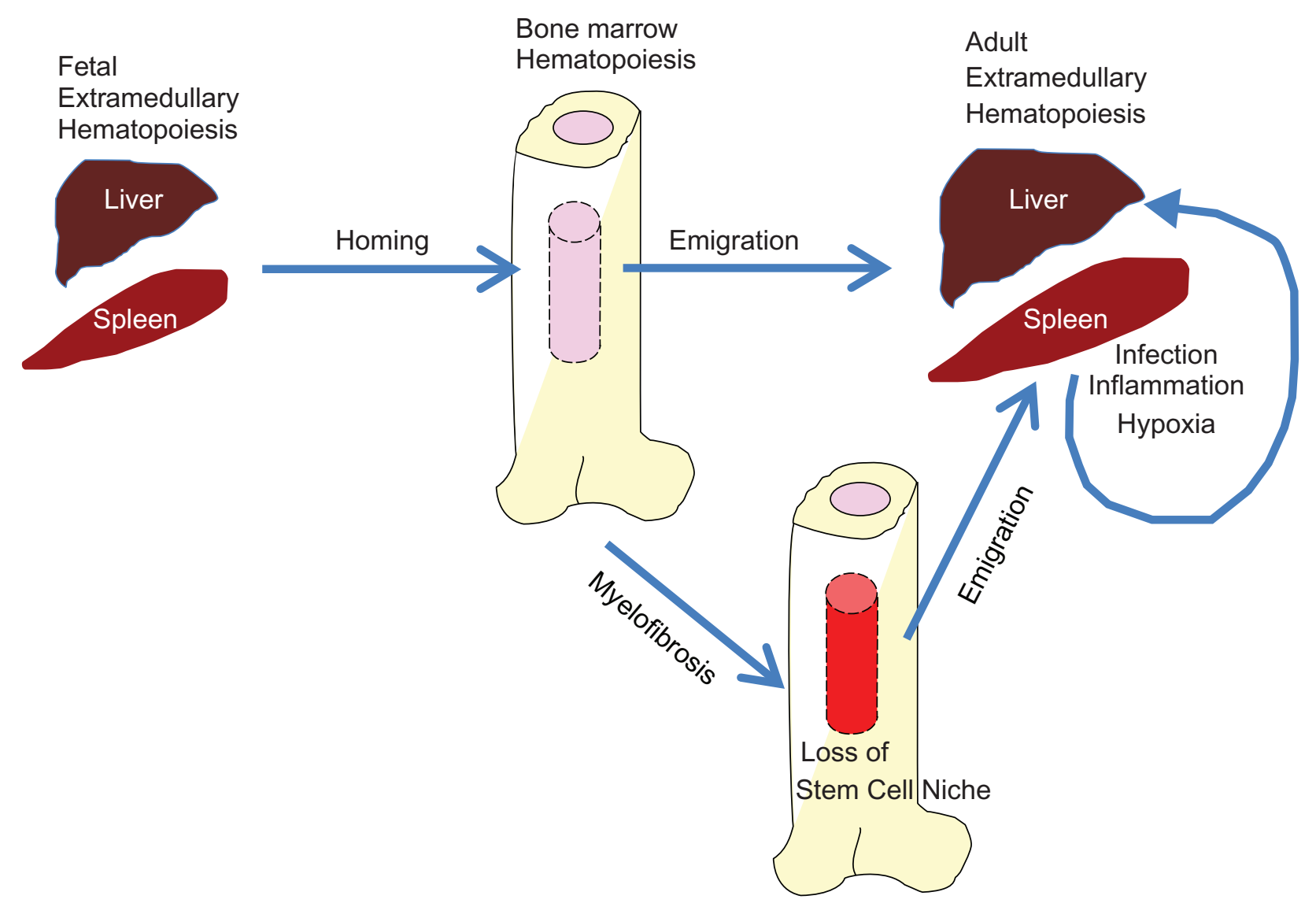

Figure I Major forms of extramedullary hematopoiesis. Extramedullary hematopoiesis occurs early in fetal development and also plays important roles in adult life. Hematopoiesis occurs in the fetal liver and spleen. Hematopoietic stem and progenitor cells in the fetal liver migrate to the bone marrow and the marrow becomes the major hematopoietic site after birth. The hematopoietic stem and progenitor cells in the bone marrow emigrate to the periphery such as the liver and spleen. Upon infection and resultant immune responses, various hematopoietic factors including TLR ligands and cytokines promote extramedullary hematopoiesis in the liver and spleen.A major role of this extramedullary hematopoiesis is to produce functionally mature antigen-presenting cells and phagocytes. Excessive and prolonged extramedullary hematopoiesis in the periphery occurs in the presence of autoimmune diseases and chronic infection. In these situations, extramedullary hematopoiesis is harmful for the host. When malignant disease such as primary myelofibrosis occurs, the marrow becomes unsuitable to support hematopoiesis and extramedullary hematopoiesis is greatly increased.

stem cell niche, which is low in oxygen and favorable to maintaining their identity as stem cells. ${ }^{2}$ The marrow stem cell niche is composed of various cells and their products which positively and negatively regulate the process. ${ }^{3,4}$ Key regulatory cells include osteoblasts, CXC chemokine ligand 12 (CXCL12)-expressing reticular cells, and vascular endothelium cells..$^{5-8}$ Moreover, signals from the sympathetic nervous system and osteoclasts regulate the hematopoietic stem cell egress from the bone marrow through regulation of a critical stem cell homing and retention factor termed CXCL12. ${ }^{9,10}$ The requirement of a specialized niche is a limiting factor for $\mathrm{EH}$ in the periphery. As a consequence, $\mathrm{EH}$ is usually limited in the periphery at specific times and under certain conditions.

A number of molecular pathways including Wnt, calciumsensing receptors, angiopoietin 1, Tie-2, and extracellular matrix components are involved in the process to finely control the stem cell niche. ${ }^{6,711-13}$ Although incompletely understood, these pathways are thought to be involved not only in the maintenance of the stem cell niche but also in renewal and differentiation of hematopoietic stem cells within the niche. Various cells produce hematopoietic cytokines such as stem cell factor (SCF), notch ligands, bone morphogenic proteins, transforming growth factor $\beta$, thrombopoietin (TPO), fibroblast growth factors, and insulin-like growth factor 2 , which maintain and regulate the primitive hematopoietic stem cells. ${ }^{14}$ Other cytokines, such as granulocyte colony stimulating factor (G-CSF), interleukin-3 (IL-3), IL-7, erythropoietin (EPO), granulocyte macrophage (GM)-CSF, and macrophage (M)-CSF, play important roles in the differentiation of hematopoietic stem and progenitor cells to committed cell lineages. ${ }^{15}$

In addition to the aforementioned cells and factors, hematopoiesis can be regulated by a number of other means, 


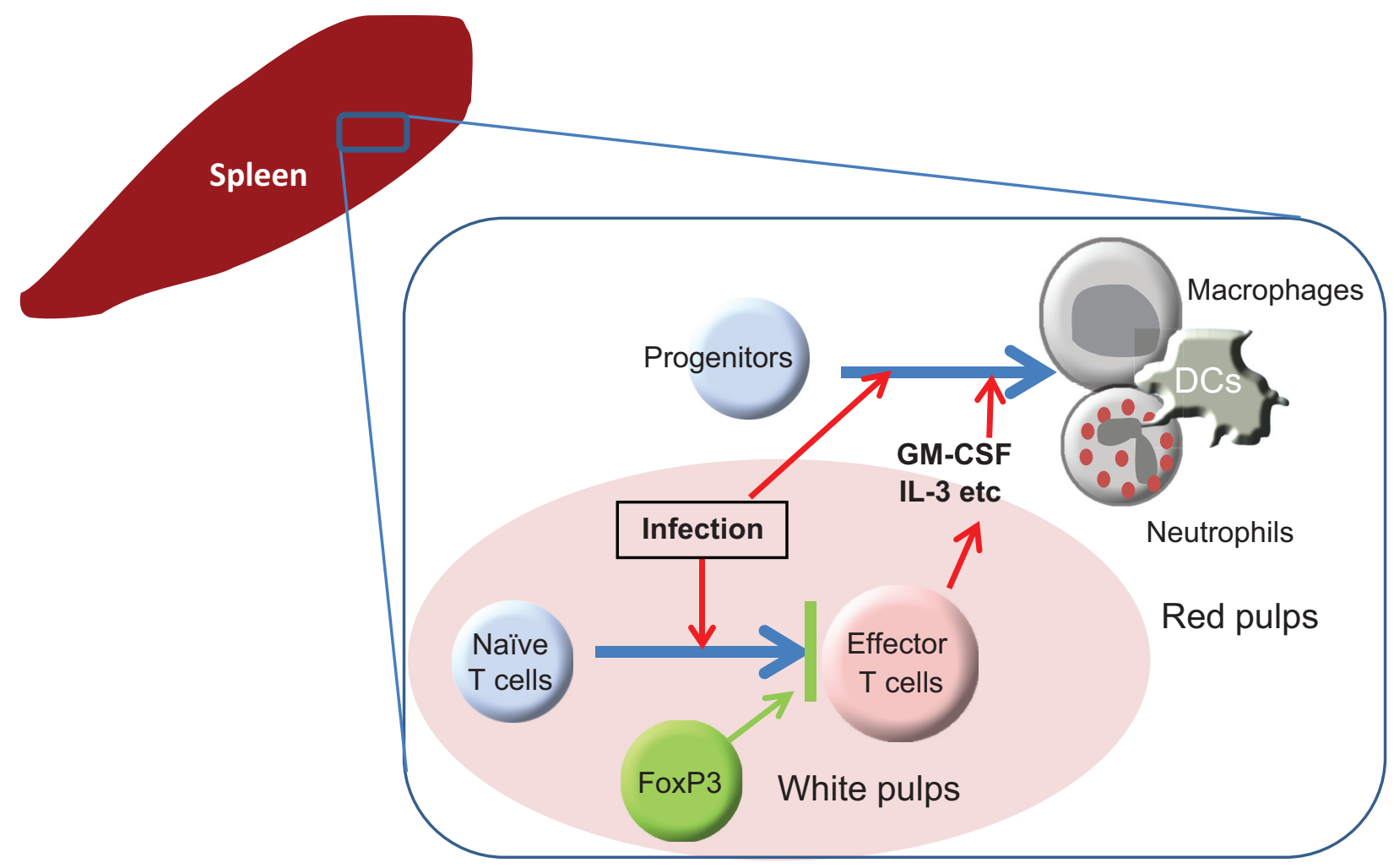

Figure 2 Regulation of extramedullary hematopoiesis during immune responses. An example of the regulatory mechanism for EH is illustrated. In the spleen, EH, exemplified by maturation of myeloid progenitors into mature neutrophils and dendritic cells, constitutively occurs at low levels. During infection and inflammation, this process is greatly increased due to activated T cells producing hematopoietic cytokines such as GM-CSF and IL-3.An important negative regulator of EH is the FoxP3 ${ }^{+}$regulatoryT cell. FoxP3 $3^{+}$regulatoryT cells suppress the differentiation of naive T cells into hematopoietic cytokine-producing effectorT cells in response to antigens imparted by antigen-presenting cells. Since it is known that FoxP3 ${ }^{+}$regulatory $\mathrm{T}$ cells can suppress more than $\mathrm{T}$ cells, there is the possibility that $\mathrm{FoxP}^{+}$regulatory $\mathrm{T}$ cells suppress EH via the regulation of additional target cell types.

such as Toll-like receptor ligands, metabolic/physiological products, various inflammatory mediators, and hormones. ${ }^{2,16-18}$ Many of the cytokines produced in inflammation act as myelopoietic factors, ${ }^{19}$ certain Toll-like receptor ligands promote myelopoiesis, ${ }^{20}$ and hypoxia is a well known inducer of erythropoiesis. ${ }^{21}$ Also, parathyroid hormone (PTH) and the insulin-like growth factors (IGF) control the hematopoietic stem cell niche; PTH can increase the number of bone marrow stem and progenitor cells and IGF can regulate the survival and expansion of hematopoietic stem and progenitor cells. ${ }^{18}$ Many of the cell types produced in the marrow, such as monocytes and B cells, require further maturation in the periphery to become fully functional immune cells. Monocytes will migrate into various tissue sites to become macrophages or dendritic cells and B cells must be activated in the periphery to become memory and plasma cells. Naïve $\mathrm{T}$ cells are made in the thymus from progenitors that originated in the marrow and they undergo further differentiation in response to antigens proffered by antigen-presenting cells. Thus, the term "extramedullary hematopoiesis" refers to a broad range of hematopoietic activities from the early stages of lineage commitment to late stages of hematopoietic cell maturation.

\section{Early EH during fetal development}

Bone marrow becomes functional as a site of hematopoiesis in the fetus from 4-5 months in human pregnancy. In mice, the marrow hematopoiesis is delayed somewhat and becomes active following birth. The developing fetus needs hematopoietic cells for the supply of oxygen and other less obvious reasons. Therefore, nonmarrow tissues serve as sites of hematopoiesis before the bone marrow takes over the role as the major hematopoietic site. In early embryo, the yolk sac serves as the primary site of hematopoiesis. ${ }^{22,23}$ During mid-gestation, yolk sac cells colonize the umbilical cord, the aorta-gonad-mesonephros (AGM) region, and subsequently the embryonic liver. The yolk sac produces hematopoietic stem cells and red blood cells initially, but later produces myeloid cells as well. ${ }^{24}$ In this regard, the yolk sac is an important place, harboring primitive erythroblasts and essentially all definitive HPC. Later in embryonic development, some of these cells seed the fetal liver. ${ }^{25}$ As mentioned, the 
next hematopoietic tissue site after the yolk sac is AGM and later, the fetal liver becomes a major hematopoietic site. Ultimately, the bone marrow becomes the predominant site of hematopoiesis. Following birth, the number of colonyforming hematopoietic progenitors in the spleen increases, peaking at two weeks of age in mice. ${ }^{26}$ In fact, the spleen remains a hematopoietic organ in mice throughout their lives, albeit at low levels.

It is thought that the liver ceases to act as a site of hematopoiesis after birth. However, the liver maintains hematopoietic stem cells, erythropoiesis, and myelopoiesis at low levels during adult life. ${ }^{27}$ Moreover, the liver is considered a maturation site for unconventional $\mathrm{T}$ cells including NKT cells, CD8 $\alpha \alpha$ T cells, CD4-CD8 ${ }^{-}$double-negative T cells, and $\gamma \delta \mathrm{T}$ cells.

\section{Induced EH occurs during infection and immune responses}

Following birth in mammals and rodents, extramedullary hematopoiesis occurs when immune responses occur in the periphery (Figure 1). The liver and spleen are the main sites of extramedullary hematopoiesis. Other organs such as the lungs, kidney, and the peritoneal cavity can also become the sites of hematopoiesis when in diseased states.

The increased extramedullary hematopoiesis in the spleen and liver of FAS (CD95)-deficient mice provides a useful insight into the cause of EH. ${ }^{28}$ In FAS-deficient mice, hematopoietic cells are resistant to apoptosis, allowing these cells to grow in organs which are normally not conducive for growth and differentiation of hematopoietic stem and progenitor cells. Also, immune cells are greatly expanded in these mice, producing large volumes of cytokines that can feed the growth of hematopoietic progenitor cells in the periphery. Another example of induced EH is the increased myelopoiesis found in many organs of FoxP3-null scurfy mice. Scurfy mice display increased $\mathrm{CD} 11 \mathrm{~b}^{+}$myeloid cells in the spleen and the liver, which is consistent with increased expression of GM-CSF and IL-3. ${ }^{29}$ In scurfy mice, T cells producing GM-CSF and IL-3 are overly activated in the absence of FoxP3 ${ }^{+} \mathrm{T}$ cells, driving excessive $\mathrm{EH}$ in the spleen and liver. By contrast, the transfer of functional FoxP3 ${ }^{+}$ $\mathrm{T}$ cells into newborn scurfy mice completely suppresses $\mathrm{EH}$ and scurfy-related inflammatory disease.$^{30} \mathrm{An}$ important function of the FoxP3 ${ }^{+} \mathrm{T}$ cells is to suppress the differentiation of naïve $\mathrm{T}$ cells into hematopoietic cytokine-producing $\mathrm{T}$ effector T cells (Figure 2).$^{30}$ FoxP $3^{+} \mathrm{T}$ cells function mainly in the $\mathrm{T}$-ell zone of the spleen rather than in the red pulps where EH occurs.
NK cells appear to play a negative role in the regulation of EH in the spleen. Depletion of NK cells with the anti-NK1.1 antibody in postnatal mice increased myeloid progenitor cells by $3-10$ times in the spleen. ${ }^{31}$ This study, however, did not determine if the increase was the outcome of cell mobilization from the bone marrow or the product of the $\mathrm{EH}$ in the spleen. In vitro, NK cells can conversely decrease the numbers of progenitor cells. ${ }^{32,33}$ While the mechanism remains to be determined, NK cells could perhaps regulate the hematopoiesis with soluble inhibitory factors or through their cell-killing activity.

Toxic shock syndrome toxin-1 (TSST-1) is a superantigen produced by most Staphylococcus aureus strains. Superantigens can conjugate the MHC II molecules of antigenpresenting cells and certain $\beta$ chains of $\mathrm{T}$ cell receptors for polyclonal activation of $\mathrm{T}$ cells. $\mathrm{T}$ cells are a good source of hematopoietic factors such as GM-CSF, IL-3 and oncostatin M..$^{30,34,35}$ In this regard, TSST-1 can induce the production of hemopoietic factors. ${ }^{36}$ Staphylococcus enterotoxin B (SEB) is another superantigen produced by Staphylococcus aureus. SEB can activate naïve $T$ cells and induce their differentiation into effector T cells that produce GM-CSF and IL-3. ${ }^{30}$

Microbial components such as TLR ligands can affect hematopoiesis in both the marrow and spleen. TLR ligands such as lipopolysaccharides (LPS, a TLR2 ligand) and Pam3CSK4 (a TLR4 ligand) can directly activate hematopoietic progenitor cells through their receptors. ${ }^{37}$ Consequently, differentiation of progenitors into myeloid cells, such as macrophages and dendritic cells, is greatly enhanced in vitro while myeloid cells in marrow and spleen are increased following the injection of LPS in vivo. Interestingly, TLR ligands can turn even lymphoid progenitors into myeloid cells. Thus, TLR ligands are effective regulators of extramedullary hematopoiesis. ${ }^{37}$ This suggests that infection may significantly affect the hematopoiesis in the marrow and peripheral organs, such as the spleen.

The function of TLR ligands in regulation of hematopoiesis suggests that infection is an important element for EH to occur. As an example, it has been determined that Leishmania major infection increases colony-forming unit cells or myeloid progenitors in the spleen..$^{38}$ Interestingly, the response in $\mathrm{BALB} / \mathrm{c}$ mice was greater than that in $\mathrm{C} 57 \mathrm{BL} / 6$ mice. This could be due to the lesser ability of BALB/c mice to clear the infection by intracellular pathogens. One of the mechanisms for the increased myelopoietic activity appears to be the production of GM-CSF and TNF- $\alpha,{ }^{39}$ which can synergistically support the growth and maturation of myeloid progenitors. 
Infection with Plasmodium berghei (ie, malaria) provided more insights into the kinetics of hematopoietic shift from the bone marrow to spleen. ${ }^{40}$ Infection with Plasmodium berghei increased numbers of CFU-GM in the bone marrow during the first week of infection, which was followed by a rise of CFU-GM in the peripheral blood and finally in the spleen around the end of the second week. The marrow progenitor numbers were normalized within two weeks, but those of the spleen remained elevated for a more prolonged time period. It is unclear how the hematopoiesis in the marrow and the spleen is sequentially regulated.

$\mathrm{EH}$ in an infection would generate sufficient numbers of mature myeloid cells to help clear pathogens, however, increased myelopoiesis without proper maturation of the myeloid progenitors could induce $\mathrm{CD} 11 \mathrm{~b}^{+} \mathrm{GR}-1^{+}$myeloidderived suppressor cells. ${ }^{41,42}$ Myeloid-derived suppressor cells emerge in the spleen and other organs impacted by infection and cancer. These cells are highly heterogeneous and are considered normal constituents of myelopoiesis. ${ }^{41}$ Thus, incomplete EH could ultimately hamper immune responses to pathogens.

\section{EH as the result of chronic myeloproliferative syndrome}

Extramedullary hematopoiesis occurs if the bone marrow is no longer functional. Primary myelofibrosis is a form of Philadelphia-negative chronic myeloproliferative syndrome. ${ }^{43}$ In primary myelofibrosis, displacement and mobilization of stem and progenitor cells occur. As a consequence, hematopoietic stem and progenitor cells occupy the liver and spleen as alternative sites of hematopoiesis. At the same time, the bone marrow stem cell niche is altered so that it no longer supports normal hematopoiesis. ${ }^{44-46}$ Thus, in primary myelofibrosis, the site of hematopoiesis changes from the marrow to the spleen and liver. Primary myelofibrosis and related Philadelphia-negative chronic myeloproliferative syndrome are associated with a point mutation in the tyrosine kinase JAK2 (JAK2V617F). ${ }^{47-50}$ It is thought that this mutation makes hematopoietic stem and progenitor cells more sensitive to growth factors, alters the marrow stem cell niche, and causes the cells to mobilize to the spleen and liver. Another notable feature of primary myelofibrosis is the high production of inflammatory cytokines such as SDF-1, HGF, IL-6, IL-8, SCF, and VEGF. Likewise, the factors that promote fibrosis and angiogenesis (bFGF, TGF- $\beta$, PF4, VEGF, etc) are increased. ${ }^{51-53}$ These molecules are produced mainly by hematopoietic cells and contribute to the regulatory humoral changes occurring within the medullar and spleen niches.
In addition to the JAK2 mutation, activating mutations affecting the thrombopoietin receptor MPL (MPLW515L and MPLW515K) have been found in small numbers of patients with Philadelphia-negative chronic myeloproliferative syndrome. ${ }^{54,55} \mathrm{EH}$, particularly $\mathrm{EH}$ in myelofibrosis, can never fully replace the marrow hematopoiesis in production of necessary blood cells, however.

\section{Conclusion}

While the bone marrow is the major site of hematopoiesis, it can occur in many other tissues both during fetal development and after birth. Extramedullary hematopoiesis can occur as long as there are appropriate supporting cells, accommodation of hematopoietic progenitors, and local production of soluble and cell-bound hematopoietic factors that maintain and induce differentiation of the stem and progenitor cells. Extramedullary hematopoiesis occurs under several conditions, both actively and passively. It plays an essential role during fetal development, namely the survival of the fetus before formation of the functional marrow hematopoietic niche. As a normal response to infection and inflammation, myelopoiesis occurs in the spleen and liver to produce phagocytic cells and antigen-presenting cells. In malignant conditions, such as various forms of myelofibrosis, the marrow hematopoietic niche becomes inhabitable, and hematopoietic stem and progenitor cells move out to the periphery. EH cannot fully supplant the marrow hematopoiesis in terms of the production of necessary hematopoietic cells, rather it is imperative for the maturation of hematopoietic cells which are produced as immature cells in the marrow. While programmed extramedullary hematopoiesis is required to supplement the hematopoietic activity in the bone marrow, excessive and disease-associated extramedullary hematopoiesis can occur and mediate chronic inflammation. More research is required to discover useful strategies for controlling unwanted extramedullary hematopoiesis and chronic inflammation.

\section{Acknowledgments}

I thank Jeeho Lee in the laboratory of Immunology and Hematopoiesis for sharing his insights. This work was supported, in part, from grants from NIH (R01AI074745, 1R56AI080769, and R01DK076616), the Crohn's and Colitis Foundation of America, and the American Heart Association to $\mathrm{CHK}$.

\section{Disclosure}

The author reports no conflicts of interest in this work. 


\section{References}

1. Bozzini CE, Barrio Rendo ME, Devoto FC, Epper CE. Studies on medullary and extramedullary erythropoiesis in the adult mouse. $\mathrm{Am} \mathrm{J}$ Physiol. 1970;219(3):724-728.

2. Eliasson P, Jonsson JI. The hematopoietic stem cell niche: Low in oxygen but a nice place to be. J Cell Physiol. 2010;222(1):17-22.

3. Garrett RW, Emerson SG. Bone and blood vessels: the hard and the soft of hematopoietic stem cell niches. Cell Stem Cell. 2009;4(6):503-506.

4. Raaijmakers MH, Scadden DT. Evolving concepts on the microenvironmental niche for hematopoietic stem cells. Curr Opin Hematol. 2008;15(4):301-306.

5. Calvi LM, Adams GB, Weibrecht KW, et al. Osteoblastic cells regulate the haematopoietic stem cell niche. Nature. 2003;425(6960):841-846.

6. Sugiyama T, Kohara H, Noda M, Nagasawa T. Maintenance of the hematopoietic stem cell pool by CXCL12-CXCR4 chemokine signaling in bone marrow stromal cell niches. Immunity. 2006;25(6):977-988.

7. Zhang J, Niu C, Ye L, et al. Identification of the haematopoietic stem cell niche and control of the niche size. Nature. 2003;425(6960):836-841.

8. Kiel MJ, Yilmaz OH, Iwashita T, Yilmaz OH, Terhorst C, Morrison SJ. SLAM family receptors distinguish hematopoietic stem and progenitor cells and reveal endothelial niches for stem cells. Cell. 2005;121(7):1109-1121.

9. Katayama Y, Battista M, Kao WM, et al. Signals from the sympathetic nervous system regulate hematopoietic stem cell egress from bone marrow. Cell. 2006;124(2):407-421.

10. Kollet O, Dar A, Shivtiel S, et al. Osteoclasts degrade endosteal components and promote mobilization of hematopoietic progenitor cells. Nat Med. 2006;12(6):657-664.

11. Arai F, Hirao A, Ohmura M, et al. Tie2/angiopoietin-1 signaling regulates hematopoietic stem cell quiescence in the bone marrow niche. Cell. 2004;118(2):149-161.

12. Nilsson SK, Johnston HM, Whitty GA, et al. Osteopontin, a key component of the hematopoietic stem cell niche and regulator of primitive hematopoietic progenitor cells. Blood. 2005;106(4):1232-1239.

13. Stier S, Ko Y, Forkert R, et al. Osteopontin is a hematopoietic stem cell niche component that negatively regulates stem cell pool size. $J$ Exp Med. 2005;201(11):1781-1791.

14. Zhang CC, Lodish HF. Cytokines regulating hematopoietic stem cell function. Curr Opin Hematol. 2008;15(4):307-311.

15. Bronchud MH, Dexter TM. Clinical use of haematopoietic growth factors. Blood Rev. 1989;3(1):66-70.

16. McGettrick AF, O’Neill LA. Toll-like receptors: key activators of leucocytes and regulator of haematopoiesis. Br J Haematol. 2007; 139(2):185-193.

17. Sioud M, Floisand Y, Forfang L, Lund-Johansen F. Signaling through toll-like receptor $7 / 8$ induces the differentiation of human bone marrow CD34+ progenitor cells along the myeloid lineage. J Mol Biol. 2006; 364(5):945-954.

18. Garrett RW, Emerson SG. The role of parathyroid hormone and insulin-like growth factors in hematopoietic niches: physiology and pharmacology. Mol Cell Endocrinol. 2008;288(1-2):6-10.

19. Watanabe S, Nakayama N, Yokota T, Arai K, Miyajima A. Colonystimulating factors and cytokine receptor network. Curr Opin Biotechnol. 1991;2(2):227-237.

20. Pevsner-Fischer M, Morad V, Cohen-Sfady M, et al. Toll-like receptors and their ligands control mesenchymal stem cell functions. Blood. 2007;109(4):1422-1432.

21. Adamson JW. Erythropoietin: in vitro and in vivo studies of the regulation of erythropoiesis. Soc Gen Physiol Ser. 1988;43:57-65.

22. Palis J, Yoder MC. Yolk-sac hematopoiesis: the first blood cells of mouse and man. Exp Hematol. 2001;29(8):927-936.

23. Tavassoli M. Embryonic and fetal hemopoiesis: an overview. Blood Cells. 1991;17(2):269-281; discussion 282-286.

24. Samokhvalov IM, Samokhvalova NI, Nishikawa S. Cell tracing shows the contribution of the yolk sac to adult haematopoiesis. Nature. 2007;446(7139):1056-1061.
25. Lux CT, Yoshimoto M, McGrath K, Conway SJ, Palis J, Yoder MC. All primitive and definitive hematopoietic progenitor cells emerging before E10 in the mouse embryo are products of the yolk sac. Blood. 2008;111(7):3435-3438.

26. Wolber FM, Leonard E, Michael S, Orschell-Traycoff CM, Yoder MC, Srour EF. Roles of spleen and liver in development of the murine hematopoietic system. Exp Hematol. 2002;30(9):1010-1019.

27. Golden-Mason L, O'Farrelly C. Having it all? Stem cells, haematopoiesis and lymphopoiesis in adult human liver. Immunol Cell Biol. 2002; 80(1):45-51.

28. Schneider E, Moreau G, Arnould A, et al. Increased fetal and extramedullary hematopoiesis in Fas-deficient C57BL/6-1pr/lpr mice. Blood. 1999;94(8):2613-2621.

29. Clark LB, Appleby MW, Brunkow ME, Wilkinson JE, Ziegler SF, Ramsdell F. Cellular and molecular characterization of the scurfy mouse mutant. J Immunol. 1999;162(5):2546-2554.

30. Lee J H, Wang C, Kim $\mathrm{CH}$. FoxP3 ${ }^{+}$regulatory T cells restrain splenic extramedullary myelopoiesis via suppression of hemopoietic cytokineproducing T cells. J Immunol. 2009;183(10):6377-6386.

31. Hansson M, Petersson M, Koo GC, Wigzell H, Kiessling R. In vivo function of natural killer cells as regulators of myeloid precursor cells in the spleen. Eur J Immunol. 1988;18(3):485-488.

32. Holmberg LA, Miller BA, Ault KA. The effect of natural killer cells on the development of syngeneic hematopoietic progenitors. J Immunol. 1984;133(6):2933-2939.

33. Degliantoni G, Perussia B, Mangoni L, Trinchieri G. Inhibition of bone marrow colony formation by human natural killer cells and by natural killer cell-derived colony-inhibiting activity. J Exp Med. 1985;161(5):1152-1168.

34. Mosmann TR, Cherwinski H, Bond MW, Giedlin MA, Coffman RL. Two types of murine helper T cell clone. I. Definition according to profiles of lymphokine activities and secreted proteins. J Immunol. 1986;136(7):2348-2357.

35. Broxmeyer HE, Bruns HA, Zhang S, et al. Th1 cells regulate hematopoietic progenitor cell homeostasis by production of oncostatin M. Immunity. 2002;16(6):815-825.

36. Galelli A, Anderson S, Charlot B, Alouf JE. Induction of murine hemopoietic growth factors by toxic shock syndrome toxin-1. J Immunol. 1989;142(8):2855-2863.

37. Nagai Y, Garrett KP, Ohta S, et al. Toll-like receptors on hematopoietic progenitor cells stimulate innate immune system replenishment. Immunity. 2006;24(6):801-812.

38. Mirkovich AM, Galelli A, Allison AC, Modabber FZ. Increased myelopoiesis during Leishmania major infection in mice: generation of "safe targets', a possible way to evade the effector immune mechanism. Clin Exp Immunol. 1986;64(1):1-7.

39. Cotterell SE, Engwerda CR, Kaye PM. Leishmania donovani infection of bone marrow stromal macrophages selectively enhances myelopoiesis, by a mechanism involving GM-CSF and TNF-alpha. Blood. 2000; 95(5):1642-1651

40. Mungyer G, Poels LG, Jerusalem C, Jerusalem R. Plasmodium berghei: influence on granulopoiesis and macrophage production in BALB/c mice. Exp Parasitol. 1983;56(2):266-276.

41. Ostrand-Rosenberg S, Sinha P. Myeloid-derived suppressor cells: linking inflammation and cancer. J Immunol. 2009;182(8):4499-4506.

42. Gabrilovich DI, Nagaraj S. Myeloid-derived suppressor cells as regulators of the immune system. Nat Rev Immunol. 2009;9(3):162-174.

43. Abdel-Wahab OI, Levine RL. Primary myelofibrosis: update on definition, pathogenesis, and treatment. Annu Rev Med. 2009;60:233-245.

44. Thiele J, Steinberg T, Hoeppner B, et al. Histo- and immunomorphometry of megakaryopoiesis in chronic myeloid leukemia with myelofibrosis and so-called primary (idiopathic) osteo-myelofibrosis/-sclerosis. Anal Cell Pathol. 1990;2(4):215-227.

45. Thiele J, Rompcik V,Wagner S, Fischer R. Vascular architecture and collagen type IV in primary myelofibrosis and polycythaemia vera: an immunomorphometric study on trephine biopsies of the bone marrow. Br J Haematol. 1992;80(2):227-234. 
46. Pereira A, Cervantes F, Brugues R, Rozman C. Bone marrow histopathology in primary myelofibrosis: clinical and haematologic correlations and prognostic evaluation. Eur J Haematol. 1990; 44(2):95-99.

47. Wernig G, Mercher T, Okabe R, Levine RL, Lee BH, Gilliland DG. Expression of Jak2V617F causes a polycythemia vera-like disease with associated myelofibrosis in a murine bone marrow transplant model. Blood. 2006;107(11):4274-4281.

48. Levine RL, Wadleigh M, Cools J, et al. Activating mutation in the tyrosine kinase JAK2 in polycythemia vera, essential thrombocythemia, and myeloid metaplasia with myelofibrosis. Cancer Cell. 2005;7(4):387-397.

49. Levine RL, Belisle C, Wadleigh M, et al. X-inactivation-based clonality analysis and quantitative JAK2V617F assessment reveal a strong association between clonality and JAK2V617F in PV but not ET/MMM, and identifies a subset of JAK2V617F-negative ET and MMM patients with clonal hematopoiesis. Blood. 2006;107(10):4139-4141.

50. Lacout C, Pisani DF, Tulliez M, Gachelin FM, Vainchenker W, Villeval JL. JAK2V617F expression in murine hematopoietic cells leads to MPD mimicking human PV with secondary myelofibrosis. Blood. 2006;108(5):1652-1660.
51. Le Bousse-Kerdiles MC, Martyre MC. Dual implication of fibrogenic cytokines in the pathogenesis of fibrosis and myeloproliferation in myeloid metaplasia with myelofibrosis. Ann Hematol. 1999;78(10):437-444.

52. Lataillade JJ, Pierre-Louis O, Hasselbalch HC, et al. Does primary myelofibrosis involve a defective stem cell niche? From concept to evidence. Blood. 2008;112(8):3026-3035.

53. Emadi S, Clay D, Desterke C, et al. IL- 8 and its CXCR1 and CXCR2 receptors participate in the control of megakaryocytic proliferation, differentiation, and ploidy in myeloid metaplasia with myelofibrosis. Blood. 2005;105(2):464-473.

54. Pikman Y, Lee BH, Mercher T, et al. MPLW515L is a novel somatic activating mutation in myelofibrosis with myeloid metaplasia. PLoS Med. 2006;3(7):e270.

55. Pardanani AD, Levine RL, Lasho T, et al. MPL515 mutations in myeloproliferative and other myeloid disorders: a study of 1182 patients. Blood. 2006;108(10):3472-3476.
Journal of Blood Medicine

\section{Publish your work in this journal}

The Journal of Blood Medicine is an international, peer-reviewed, open access, online journal publishing laboratory, experimental and clinical aspects of all topics pertaining to blood based medicine including but not limited to: Transfusion Medicine; Blood collection, Donor issues, Transmittable diseases, and Blood banking logistics; Immunohematology; Artificial and alternative blood

\section{Dovepress}

based therapeutics; Hematology; Biotechnology/nanotechnology of blood related medicine; Legal aspects of blood medicine; Historical perspectives. The manuscript management system is completely online and includes a very quick and fair peer-review system. Visit http://www.dovepress.com/ testimonials.php to read real quotes from published authors.

Submit your manuscript here: http://www.dovepress.com/Journal-of-blood-medicine-journal 